\title{
Study of Two-Dimensional Melting in a System of Small Magnets
}

\author{
M. Šviková ${ }^{a}$, M. LišČInskÝ ${ }^{a}, \mathrm{P} . \mathrm{RABATIN}^{b}, \mathrm{M} . \mathrm{GÉCI}^{a}$ \\ ${ }^{a}$ High School of st. Thomas Aquinas, Zbrojničná 3, Košice 040 01, Slovakia \\ ${ }^{b}$ Faculty of Electrical Engineering and Informatics, Technical University of Košice, Letná 9, 04200 Košice, Slovakia

\begin{abstract}
Using video tracking we study configurational and melting properties of a finite number (64) of 6 mm sized disc magnets moving on an air layer produced by an air table to imitate two-dimensional (2D) crystal. It is shown that velocity of air flow allows us to influence temperature. Analysing trajectories of particles has shown the transition from hexagonal to square lattice and after it to liquid phase with underlying square shell structure.
\end{abstract}

DOI: $10.12693 /$ APhysPolA.126.258

PACS: 64.70.D-, 64.70.dj, 68.90.+g

\section{Introduction}

The effort to understand the microscopic mechanism of melting has intrigued scientists for more than 100 years, but many questions about melting mechanisms remain to be answered [1]. Dimensionality, for example, plays an important role in melting; three-dimensional (3D) crystals directly melt into a liquid phase via a first-order transition, but the nature of two-dimensional (2D) crystal melting has been richly debated for decades [2-5]. In the popular Kosterlitz-Thouless-Halperin-Nelson-Young (KTHNY) scenario, 2D crystals first melt into a hexatic phase and then melt from the hexatic phase to a liquid phase via two continuous phase transitions associated with the creation of dislocations and disclinations [2]. On the other side the experimental and computer simulations studies [6-8] of structural and dynamical properties of small classical 2D clusters confined in circular confinement found that the particles are arranged in shells and and that melting of a finite clusters is a two-step process. With increasing temperature, intershell motion develops and the system loses angular order. After that, radial diffusion starts and destroys the shell structure of the cluster .

In this paper we study the system of a finite number of small cylindrical magnets moving on an air layer in which melting behavior of $2 \mathrm{D}$ system can be investigated.

\section{Experimental system}

We place 64 disc magnets (weight: $0.67 \mathrm{~g}, 6 \mathrm{~mm}$ in diameter and $1 \mathrm{~mm}$ high, NdFeB, N45) on plexiglass pad of air table. Magnetic moments of magnets are oriented perpendicularly to the layer. Magnets repel each other by dipolar magnetic interaction. Square magnetic edge with side of $22.3 \hat{A} Z \mathbf{z} \mathrm{cm}$ magnetically repulses the discs. Magnets are moving freely on an air layer. We can influence their velocities by changing the velocity of air current blown to the air table (Fig. 1). We record the system

corresponding author; e-mail: svikova@gmail.com with a webcam, with frame rate $30 \mathrm{fps}$. We trace trajectories of each magnet (particle) by analyzing video with particle recognition algorithm. Then we calculate velocities of the magnets from their displacement and time interval between two images.

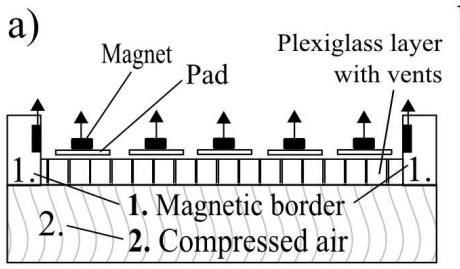

b)

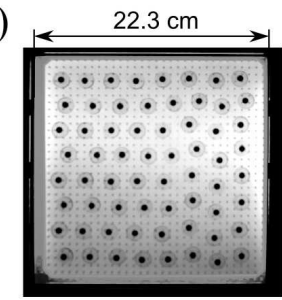

Fig. 1. a) Schematic side view of the air table, b) Photograph (top view) of system of magnets.

\section{Results and discussion}

In our experiment the current of air gives the magnets kinetic energy, a method which differs from the natural process of heating and is similar to mechanic shaking used in [5]. Therefore we must verify if the velocities of the magnets are of a Maxwellian distribution in order to simulate the melting process properly. We define the reduced temperature $\tau=\sum\left(v_{i}^{2}\right) / N$, where $v_{i}$ is velocity of i-the particle, $N$ is the number of particles.
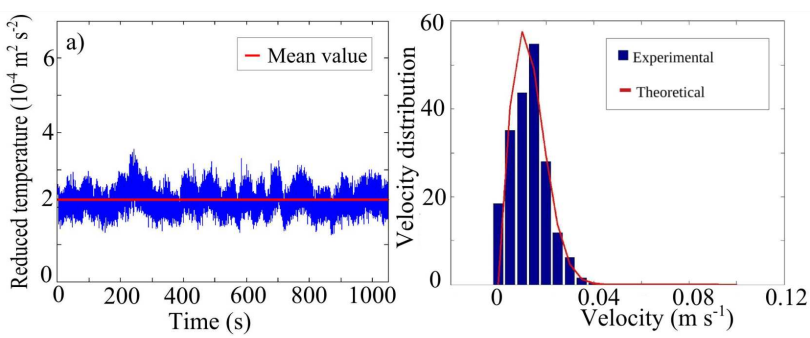

Fig. 2. a) Fluctuations of reduced temperature with time. b) Theoretical Maxwellian velocity distribution and experimental observation, $\tau=2.2 \times 10^{-4} \mathrm{~m}^{2} \mathrm{~s}^{-2}$. 
$\tau=0.5$

a)

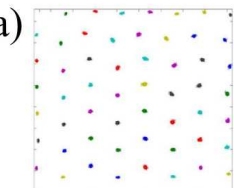

b)

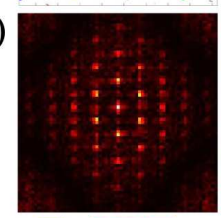

c)

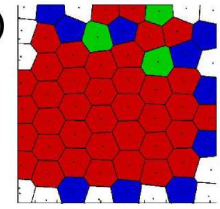

2.2
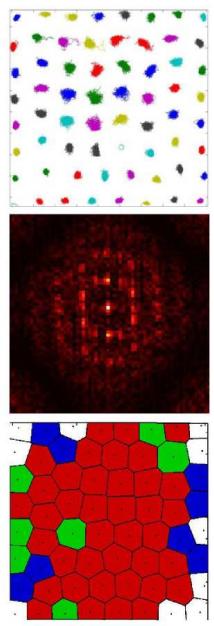

12.9

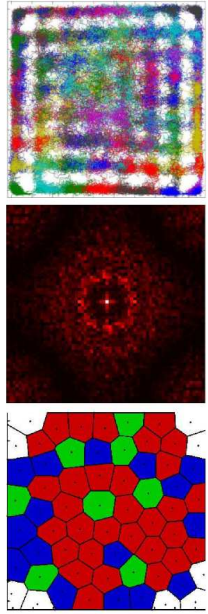

Fig. 3. a) Traces of magnets during $5 \times 10^{3}$ time steps. b) Numerically generated diffractograms. c) Voronoi diagrams (indicated $\langle\tau\rangle$ is in units $10^{-4} \mathrm{~m}^{2} \mathrm{~s}^{-2}$ ).

Figure 3a) shows the particle trajectories for different temperatures and in Fig. 3b) there are depicted diffractograms generated numerically using fast Fourier transform similarly as in [5]. 2D hexagonal lattice is formed at low temperature and particles oscillate around their equilibrium positions. The scattering pattern is composed of discrete bright spots in a hexagonal array. With the increase of the temperature particles exhibit larger amplitude of motion. In plot of trajectories at $\langle\tau\rangle=2.2 \times 10^{-4} \mathrm{~m}^{2} \mathrm{~s}^{-2}$ we can notice the change of the symmetry of the lattice - from hexagonal to square lattice. It can be seen also in diffractogram in Fig. 3b). Further increase of the temperature causes the movement of particles around the whole system area and despite of that trajectories create underlying square lattice with regions of larger particle presence probability. We suppose that it reveals a similar phenomenon as in circular shell structure described in $[6,8]$. Particularly the particle diffusion arises first in the square shells and inter shell diffusion is less probable. As picture of trajectories shows, this hopping process is more probable in the middle of the system area than near the border. The diffractogram is in this case reduced to a few rings without the bright spots which indicates the liquid state [5].

The topological defects play a role in melting process $[2,3]$. In Voronoi diagrams shown in Fig. 3c) the defects (non-six-sided cells) are marked by green colour for sevenfold and by blue colour for fivefold coordination number. Fig. 4. a) plots the dependence of averaged number density of defects on reduced temperature. It exhibits large transition around $\langle\tau\rangle=2.210^{-4} \mathrm{~m}^{2} \mathrm{~s}^{-2}$. Fig. $4 \mathrm{~b}$ ) shows the averaged defect density distribution for two values of $\langle\tau\rangle$.

At low temperature there are geometry induced defects in outer region near the border and as temperature increases many thermal defects are generated. Defects are more likely to emerge at the border but there is also pronounce increase of the defects density on the whole system area.

Gradual loss of translational order can be seen also in Fig. 4c) where correlation function $g(r)=$ $(S / N)[n(r) / 2 \pi r \Delta r]$ is shown at several temperatures. Here $n(r)$ is a number of particles situated at distance between $r$ and $r+\Delta r$ from a given particle. N-number of particles, $S$ - area of system.
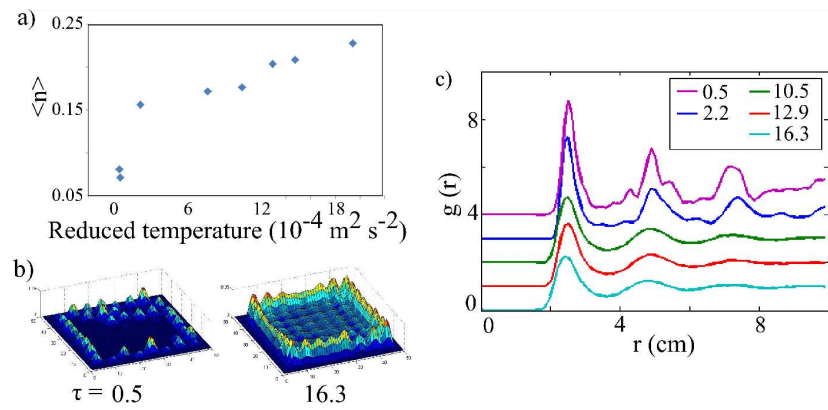

Fig. 4. a) Averaged number density of defects vs reduced temperature. b) The spatial distribution of averaged defect density. c) Correlation function $g(r)$ at indicated $\langle\tau\rangle$ in units $10^{-4} \mathrm{~m}^{2} \mathrm{~s}^{-2}$. The curves for higher $\langle\tau\rangle$ are vertically shifted.

\section{Conclusions}

We have studied the melting of two-dimensional system of small disc magnets with repulsive dipolar interaction moving on the air layer. At low temperature 2D hexagonal lattice is formed while with the increase of the temperature the change of the symmetry of the lattice from hexagonal to square is observed. The dependence of averaged number density of defects on the temperature exhibits sudden increase indicating transition to the liquid phase. Particle trajectories in this phase reveal the square shell structure with larger intra-shell diffusion and less probable inter-shell hopping.

\section{Acknowledgments}

This The work was done with support of SUSY (project APVV-LPP-0270-09).

\section{References}

[1] J.G. Dash, Rev. Mod. Phys. 71, 1737 (1999).

[2] K.J. Strandburg, Rev. Mod. Phys. 60, 161 (1988).

[3] D.E. Angelescu, C.K. Harrison, M.L. Trawick, R.A. Register, P.M. Chaikin Phys. Rev. Lett. 95, 025702 (2005).

[4] P. Keim, G. Maret, U. Hertz, H. von Grunberg, Phys. Rev. Lett. 92, 215504 (2004).

[5] X.H. Zheng, R. Grieve, Phys. Rev. B 73, 064205 (2006).

[6] R. Bubeck, C. Bechinger, S. Neser, P. Leiderer, Phys. Rev. Lett. 82, 3364 (1999).

[6] Y.-J. Lai and L. I, Phys. Rev. E 64, 015601 (2001).

[8] M. Kong, B. Partoens, F.M. Peeters, Phys. Rev. E 67, 021608 (2003). 